\title{
Flexible and Multi-Functional Graphene Sensor Platform *
}

\author{
Altynay Kaidarova ${ }^{1}$, Abeer Almoudi ${ }^{1}$, Renad M. Allagani ${ }^{1}$, Marco Marengo ${ }^{1}$, Mohammed A. Khan ${ }^{1}$, U.Buttner ${ }^{1}$ \\ Carlos M. Duarte ${ }^{2}$ and Jurgen Kosel ${ }^{1}$. \\ ${ }^{1}$ Computer, Electrical and Mathematical Sciences \& Engineering (CEMSE), King Abdullah University of Science and \\ Technology(KAUST), Thuwal, Kingdom of Saudi Arabia \\ ${ }^{2}$ Red Sea Research Center (RSRC), King Abdullah University of Science and Technology (KAUST) \\ Kingdom of Saudi Arabia, Thuwal 23955
}

jurgen.kosel@kaust.edu.

\section{SUMMARY AND MOTIVATION}

This paper presents a flexible, printed and robust sensor platform for the detection of various parameters: strain, flow speed, pressure, conductivity, temperature and magnetic field. The sensors are based on Laser-Induced Graphene fabricated by laser printing on Kapton substrate. Multi-functionality is obtained by exploiting printing process combined with the flexible nature of Kapton and the piezoresistivity of Graphene. Sensor have been implemented for the detection of strain, flow speeds, forces pressure, salinity and magnetic field. Their sensitivity and dynamic range can be tailored via the substrate and laser printing design. A robust and reliable operation was obtained during underwater experiments in the Red Sea.

\section{ADVANCES OVER PREVIOUS WORKS}

Next generation smart devices are envisioned to have enhanced electronic conductivity, thermal stability, mechanical strength, as well as affordability and amenity to mass production. The remarkable properties of graphene make this material one of the most promising candidates for such devices. Laser-induced Graphene (LIG) on flexible polyimide substrates offers an extremely attractive route for exploiting this material [1-4]. We developed a multi-functional LIG sensor platform and demonstrate its versatility by implementing underwater marine monitoring of animals' speed, current flows, and changing environmental conditions of the Red Sea.

\section{Results AND MethodOlOGY}

We utilize LIG as electrodes on a flexible substrate to develop a versatile and customizable sensor platform. Thereby, sensitivity and dynamic range can be widely tailored by simply adopting the substrate or electrode geometries. The sensors were fabricated on a polyimide film $(125 \mu \mathrm{m})$, while porous conductive carbon networks with a high content of graphene sheets $(40 \pm 3 \mu \mathrm{m})$ were photo-thermally induced by $\mathrm{CO}_{2}$ laser (Fig. 1) [1]. PDMS of $10 \mu \mathrm{m}$ thickness was spin-coated on the sensor surface to avoid biofouling and short-circuits.

For strain, flow, force and pressure sensing, the piezoresistance of the LIG electrodes was exploited. For the flow and force sensors a cantilever design is adopted and deflections induced by different forces or flow speeds are sensed. A conductivity cell is implemented with four-

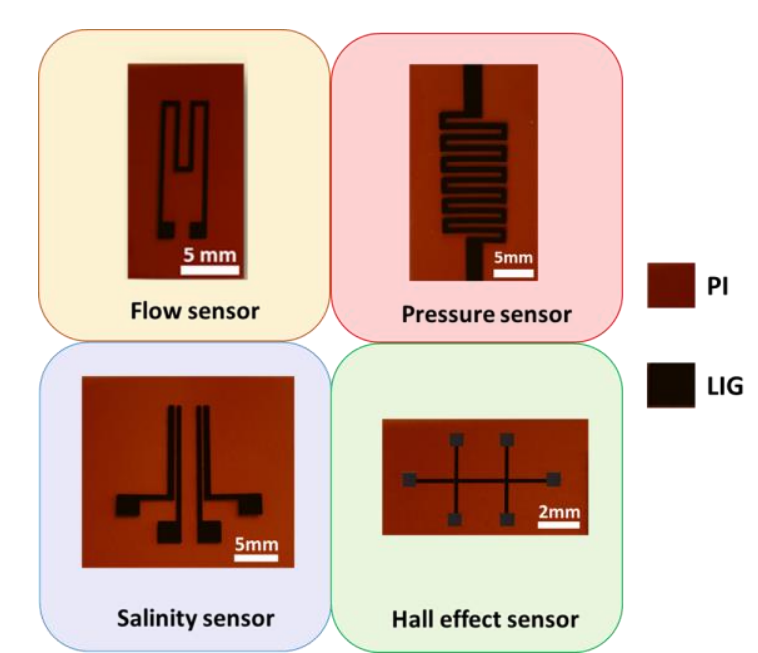

Fig. 1: Flexible sensor platform based on LIG

electrodes, where the outer electrodes drive an AC current and the inner electrodes measure the voltage drop across the medium. Together with a recently reported highly sensitive LIG thermistor [5], they pave the way to flexible and miniaturized Conductivity, Temperature and Depth (CTD) devices. An LIG magnetic field sensor is implemented as a Hall bar that converts magnetic flux density into an output voltage .

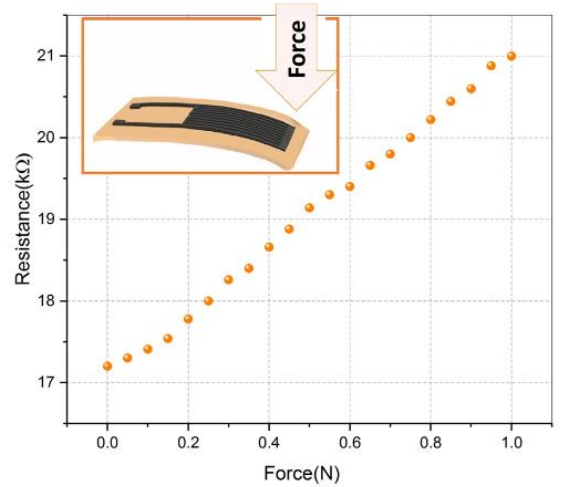

Fig. 2: Resistance as a function of force induced during extension

An electromechanical pull tester and ultrasonic flow test setup were used to test the LIG force and bending sensor. Fig.2 and Fig.3 show that the sensor has a linear relationship with the exerted force and flow with sensitivities of $\sim 4 \Omega / \mathrm{mN}$ and $\sim 300 \Omega /(\mathrm{m} / \mathrm{s})$, respectively. Upon larger deflection, saturation of the flow sensor is observed, as expected, due to the decrease in effective cross section area. The LIG sensors possess excellent 
durability at high pressures (Fig. 4), with a sensitivity of $\sim 0.1 \Omega / \mathrm{kPa}$, as well as a high resolution of $\sim 20 \mathrm{~Pa}$ (Fig.5).The conductivity cell is characterized by a sensitivity of $0.85 \mathrm{mS} / \mathrm{psu}$ and operation in different configurations, i.e.,planar or bent, over a wide range of frequencies(Fig. 6).

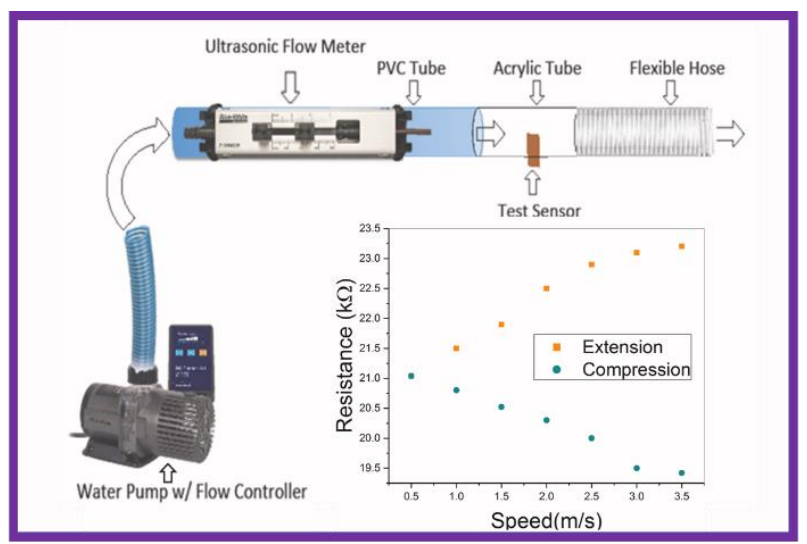

Fig. 3: Schematic of the flow test setup. The inset shows the sensor response for flow speeds from 0.5 to $3.5 \mathrm{~m} / \mathrm{s}$

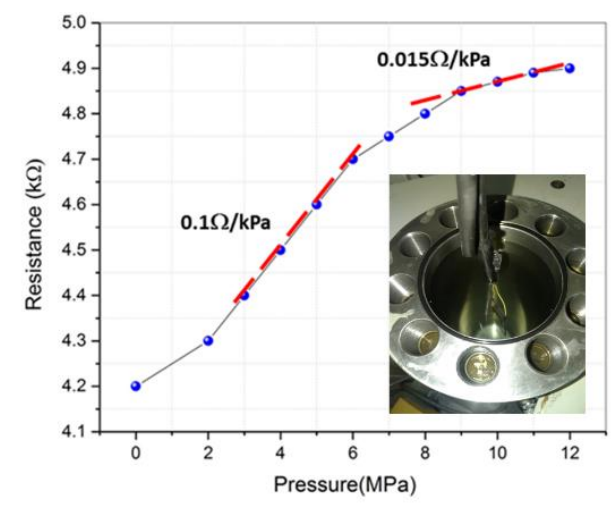

Fig. 4: Resistance response to high pressures

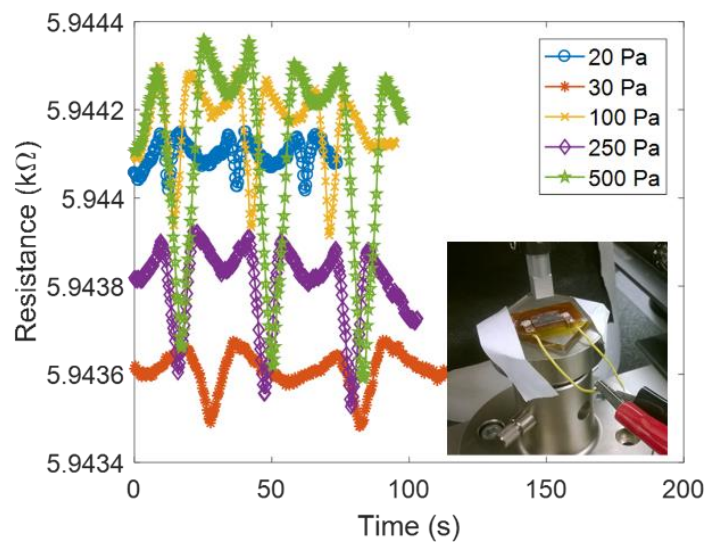

Fig. 5: Limit of detection of pressure sensors

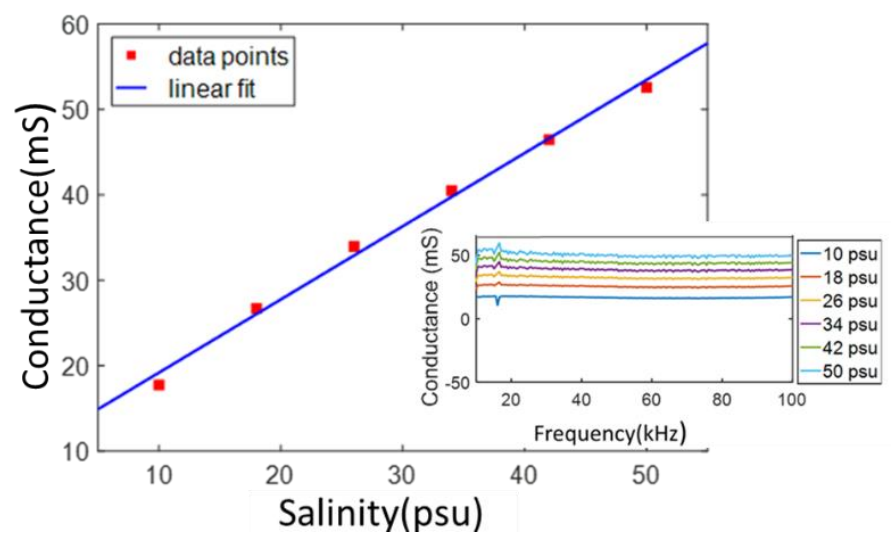

Fig. 6: The conductance as a function of salinity and the frequency.

The Hall Effect sensors have a linear response to magnetic fields with a normalized sensitivity of 1.16V/AT (Fig. 7), which showed no degradation after being bent to a minimum radius of $5 \mathrm{~mm}$. The LIG sensors were integrated with an ultra-low power aquatic tag and successfully recorded the speed of marine animals and in-situ sea parameters (Fig. 8), confirming that they can withstand harsh environmental conditions over an extended period of time. Benefiting from the versatility of the laser writing technology, LIG sensors can also be integrated into wearable electronics, highly integrated machines, soft robotics or prosthesis by tailoring the geometry.

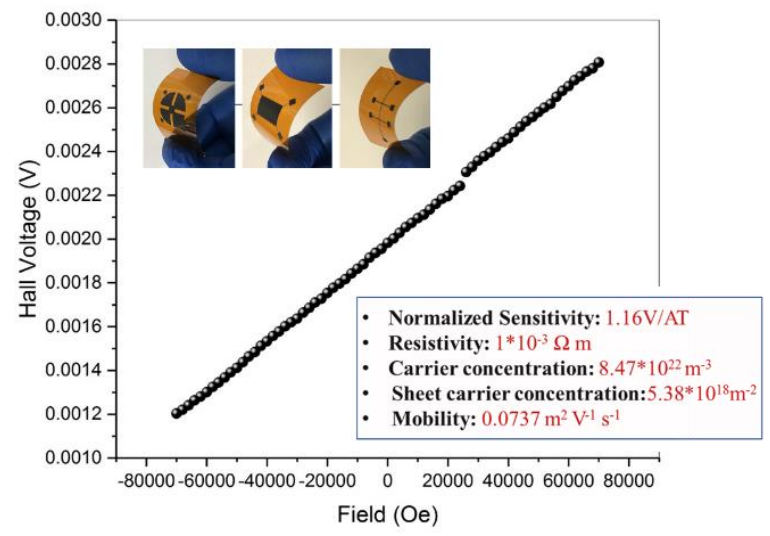

Fig. 7: Response of the LIG Hall sensor at $100 \mu \mathrm{A}$ bias current.

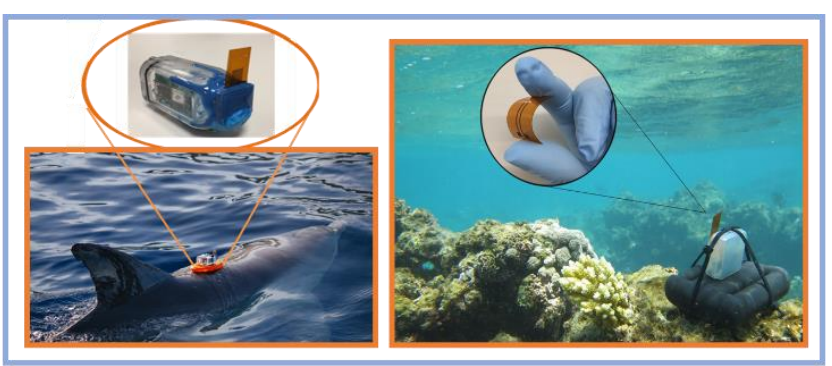

Fig. 8: Real-life applications of LIG sensors: monitoring of the speed of dolphin and oceanic properties of Red sea (left). 


\section{REFERENCES}

[1] Lin, J., Peng, Z., Liu, Y., Ruiz-Zepeda, F., Ye, R., Samuel, E.L., Yacaman, M.J., Yakobson, B.I. and Tour, J.M., 2014. Laser-induced porous graphene films from commercial polymers. Nature communications, 5, p.5714.

[2] Park, J.B., Xiong, W., Gao, Y., Qian, M., Xie, Z.Q., Mitchell, M., Zhou, Y.S., Han, G.H., Jiang, L. and Lu, Y.F., 2011. Fast growth of graphene patterns by laser direct writing. Applied Physics Letters, 98(12), p.123109..
[3] Kaidarova, A., Marengo, M., Marinaro, G., Geraldi, N., Duarte, C.M. and Kosel, J., 2018. Flexible and Biofouling Independent Salinity Sensor. Advanced Materials Interfaces, 5(23), p.1801110.

[4] Duy, L.X., Peng, Z., Li, Y., Zhang, J., Ji, Y. and Tour, J.M., 2018. Laserinduced graphene fibers. Carbon, 126, pp.472-479.

[5] Marengo, M., Marinaro, G. and Kosel, J., 2017, October.

Flexible temperature and flow sensor from laser-induced graphene. In 2017 IEEE SENSORS (pp. 1-3). IEEE. 